\title{
Shock index in patients with traumatic solid organ injury as a predictor of massive blood transfusion protocol activation
}

\author{
Ayman El-Menyar ${ }^{1,2^{*}}$ (D), Gaby Jabbour ${ }^{3}$, Mohammad Asim² $^{2}$, Husham Abdelrahman ${ }^{3}$, Ismail Mahmood ${ }^{1,3}$ and \\ Hassan Al-Thani ${ }^{4}$
}

\begin{abstract}
Purpose: We aimed to assess the utility of shock index (SI) to predict the need for massive transfusion protocol (MTP) in patients with solid organ injury (SOI) in a Level 1 Trauma center.

Methods: We conducted a retrospective analysis for patients with SOI between 2011 and 2014. Patients were categorized according to on-admission SI into low $(<0.8)$ and high SI $(\geq 0.8)$ group.

Results: A total of 4500 patients were admitted with trauma, of them 572 sustained SOls (289 patients had SI $\geq 0.8$ ). In comparison to low SI, patients with high SI were younger, had higher injury severity scores (ISS) and lower Trauma and Injury Severity Score (TRISS); $(p<0.001)$. The proportion of exploratory laparotomy (EXLap), blood transfusion (BT), MTP activation, sepsis and hospital mortality were significantly higher in patients with high SI. Serum lactate $(r$ $=0.34)$, hematocrit $(r=-0.34)$, ABC score $(r=0.62)$, ISS $(r=0.35)$, and amount of transfused blood $(r=0.22)$ were significantly correlated with SI. On multivariable regression analysis using 9 relevant variables (age, sex, ISS, ED GCS, serum lactate, hematocrit, Abdomen AIS and Focused assessment with sonography in trauma (FAST) and SI), SI $\geq 0.8$ was an independent predictor of BT (OR 2.80; 95\%Cl 1.56-4.95) and MTP (OR 2.81;95\% Cl 1.09-7.21) .
\end{abstract}

Conclusions: In patients with SOI, SI is a simple bedside predictor for BT and MTP activation. Further prospective studies are needed to support our findings.

Keywords: Shock index, Solid organ injury, Blood transfusion, Laparotomy, MTP, Trauma

\section{Introduction}

In an attempt to identify hypovolemic shock in trauma, shock index (SI) has been used as a quick bedside clinical indicator of hypovolemic shock (McNab et al. 2013). It can reliably identify hemodynamic instability (Cannon et al. 2009; Vandromme et al. 2011), and could be used for risk stratification for transfusion requirements and outcomes (Zarzaur et al. 2008). Prior studies found that prehospital and admission SI correlated with on-going bleeding and need for massive transfusion (MT) in

\footnotetext{
* Correspondence: Aymanco65@yahoo.com

This study was presented in part at the 19th Congress of the European Society for Trauma and Emergency Surgery (ESTES) held on May 6-8, 2018 at Valencia, Spain

${ }^{1}$ Clinical Medicine, Weill Cornell Medical College, Doha, Qatar

${ }^{2}$ Department of Surgery, Clinical Research, Trauma \& Vascular Surgery,

Hamad General Hospital (HGH), P.O Box 3050, Doha, Qatar

Full list of author information is available at the end of the article
}

trauma patients (Vandromme et al. 2011; Zarzaur et al. 2008; Birkhahn et al. 2005; El-Menyar et al. 2018). Polytrauma patients are frequently diagnosed to have abdominal injuries with an estimated frequency of 15-17/ 100,000 in Qatar (Arumugam et al. 2015). In particular, abdominal solid organ injury (SOI) secondary to highimpact trauma results in considerable bleeding, morbidity and mortality (El-Menyar et al. 2017). SOI includes any grade of injury to the liver, spleen, kidneys or pancreas in isolation or combination. SOI is a leading cause of mortality in trauma and its management and outcomes are mainly dependent on the patient hemodynamic stability and the early efficient control of bleeding. Timely determination of the need for MT and intervention remains challenging in patients with SOI. Rapid diagnosis of SOIs, mainly liver and spleen, is important to minimize the risk of hemorrhagic

(C) The Author(s). 2019 Open Access This article is distributed under the terms of the Creative Commons Attribution 4.0 International License (http://creativecommons.org/licenses/by/4.0/), which permits unrestricted use, distribution, and 
shock, the need for surgery and post-operative complications (Sawhney et al. 2014). Abdominal trauma patients who are found to have lower grade SOI with normal physiological status are usually treated nonoperatively (El-Menyar et al. 2017). On the other hand, hemodynamic instability despite resuscitation is an indication to consider immediate exploratory laparotomy and definitive surgery (Stawicki 2017). Also, it has been suggested that hemodynamically unstable patients or those who need $>2$ units of packed RBC transfusion following SOI require immediate laparotomy (Malhotra et al. 2002).

It has been noted that trauma patients who require MT often die within $6 \mathrm{~h}$ of the resuscitation efforts, therefore, a reliable tool to predict MT usage would be vital. There are almost 24 scoring tools and predictive models available to predict the need for MT in trauma cases. Although MT is a life-saving treatment, it can be a source of harm when it is utilized inappropriately. An optimized transfusion strategy with appropriate blood component selection is critical in the absence of the point of care testing. The resuscitative effort should start within minutes (Hsu et al. 2016), however, the compliance to MT protocols (MTP) is not optimum in reality. Bawazeer et al. found delays in 50\% of MTP activation and a $47 \%$ incidence of non-compliance with the type of blood product given (Fredericks et al. 2017). Notably, clinical gestalt is an unreliable predictor of MT with a sensitivity of only $66 \%$; it worked poorly as a screening test for MT and missed over one third of patients who ultimately required MT (El-Menyar et al. 2019). This means that trauma surgeons' threshold for MTP activation is still questionable as they missed a substantial number of cases that were potentially under resuscitated (El-Menyar et al. 2019).

Despite advancement in resuscitation, hemorrhagic shock still accounts for almost one third of all traumarelated preventable deaths (Rossaint et al. 2016). Improved prediction of significant traumatic hemorrhage may be useful for better management of blood products, and proper activation of MTP. This would reduce blood product use, and allows components of a MTP to be delivered in a timely fashion and in a high ratio [a higher fresh frozen plasma (FFP)/packed red blood cell (RBC) ratio] to treat acute traumatic coagulopathy (Khan et al. 2013).

Although SOI is one of the main sources of massive bleeding in abdominal injuries in both blunt and penetrating trauma, less is known about the prognostic implications of SI in traumatic SOI. Therefore, the current study aims to assess the utility of SI in patients with SOI, to predict the need for MTP activation, blood transfusion and exploratory laparotomy in a level I trauma center.

\section{Methods}

This was a retrospective chart review study to include all abdominal trauma patients with SOI (splenic, hepatic, renal or pancreatic injury of any grade) admitted at the level I trauma center, between June 2011 and June 2014. Relevant information was abstracted from The Qatar National Trauma Registry [QNTR] at Hamad General Hospital (HGH) after obtaining the ethical approval from the Medical Research Center (IRB\# 14409/14) at Hamad Medical Corporation. The QNTR is a database that participates in both the National Trauma Data Bank (NTDB) and the Trauma Quality Improvement Program (TQIP) of the American College of Surgeons-Committee on Trauma (ACS-COT).

Shock Index (SI) is defined as the ratio of HR to SBP on-admission at the emergency room, and we used the cutoff of 0.8 indicated for the need of MT in trauma patients as mentioned in an earlier study (El-Menyar et al. 2018). Prior study used this cutoff based on the optimum reading on the receiver operating characteristic curve (ROC). The pulse pressure (PP) was calculated as the difference between SBP and diastolic blood pressure (DBP).

We included all adult patients who were transported directly to the Emergency Department (ED) from the scene.

We excluded patients who were transferred from other hospitals, patients with prehospital cardiac arrest, and those who had incomplete data. The variables of interest were patient demographics (age \& gender), type and mechanism of injury (MOI), vital signs on admission (i. e., HR, SBP, DBP, PP), laboratory findings (lactate, hematocrit), admission Glasgow Coma scale (GCS), Injury Severity Score (ISS), Abdomen Abbreviated Injury Scale (AIS), Trauma and Injury Severity Score (TRISS), Assessment of Blood Consumption (ABC) score, site of injury (liver, spleen, kidney \& pancreas), associated injuries (head injury, hemothorax); Focused Assessment with Sonography in Trauma (FAST), intubation, exploratory laparotomy, need for blood transfusion, number of transfused packed red blood cells (PRBC) units, massive transfusion, hospital and ICU lengths of stay, ventilatory days, sepsis and mortality. SOI grading was according to the Organ Injury Scaling Committee of the American Association for the Surgery of Trauma. The American College of Surgeons has defined four classes of hypovolemic shock in the Advanced Trauma Life Support (ATLS) training program and manual; based on estimated blood loss, vital signs (blood pressure, pulse rate), and mental status. $\mathrm{ABC}$ score was calculated using 4 variables i.e. MOI (penetration $=1$, blunt $=0$ ), FAST (positive $=1$, negative $=0$ ), ED SBP $(<90 \mathrm{mmHg}=1,>90 \mathrm{mmHg}=0)$, and ED HR $(>120$ beats $/ \mathrm{min}=1,<120$ beats $/ \mathrm{min}=0)($ Schroll et al. 2018). As per the institutional protocols, Massive transfusion (MT) is defined as the replacement of the patient's total blood 
volume (approximately $5 \mathrm{l}$ ) over a $24 \mathrm{~h}$ period or actual/anticipated administration of $>40 \mathrm{~mL} / \mathrm{kg}$ PRBC in $2 \mathrm{~h}$ or less. The attending physician, trauma team leader, consultant or anesthetist is responsible for activation of the MTP. Blood bank staff will immediately prepare the first pack of blood products as 6 units of uncross-matched type $O$ positive PRBC; 6 units equivalent of platelets; and 6 units $A B$ plasma.

\section{Statistical analysis}

Data were presented as proportions, medians, or mean \pm standard deviation, as appropriate. The variables of interest were compared and analyzed according to SI in ED ( $\mathrm{SI}<0.8$ versus $\mathrm{SI} \geq 0.8$ ). The SI cutoff of 0.8 was used based on previous works (El-Menyar et al. 2018). Prior study used this cutoff based on the optimum reading on the receiver operating characteristic curve (ROC). Differences in categorical and continuous variables were analyzed using $X^{2}$ test and students t-test, as appropriate. Yates' corrected chi-square was used for categorical variables, if the expected cell frequencies were below 5 . The Pearson correlation coefficient (r) was calculated to identify the linear relationship between the SI and other relevant covariates. Predictive value of SI for MTP, blood transfusion, exploratory laparotomy and mortality was performed in terms of sensitivity, specificity, positive and negative predictive value (PPV\&NPV), positive and negative likelihood ratio (LR). Multivariable regression analyses were performed to determine the predictors of blood transfusion, MTP and exploratory laparotomy using the most relevant covariates: sex, age, ISS, abdomen AIS, ED GCS, serum lactate, hematocrit, FAST and SI (SI was used as categorical in one analysis and as a continuous variable in another analysis). For prediction of early laparotomy, MTP activation as an independent variable was added into the model in addition to the above-mentioned 8 variables. Data were expressed by the odds ratio (OR) and 95\% confidence intervals (CIs). SI was used as categorical and also as a continuous variable in the multivariable analysis. A two-tailed $P$ value of $<0.05$ was considered to be statistically significant. The Receiver Operating Characteristic (ROC) curves and area under the curves (AUC) were performed using different SI cut-offs to show the prediction power of SI for blood transfusion. All data analyses were carried out using the Statistical Package for the Social Sciences, version 18 (SPSS, Inc., Chicago, IL).

\section{Results}

During the 3-years study duration, a total of 4500 trauma patients required hospital admission, of which $572(12.7 \%)$ sustained SOIs. The mean age of patients was $29 \pm 13.0$ years and males $(89 \%)$ predominated with a male to female ratio of 8 to 1 . Blunt trauma (95\%) was most frequent injury type which constituted mainly motor vehicle crashes (78\%) and fall from height (22\%). On arrival to the trauma room, 289 (50.5\%) SOI patients had an elevated SI $(\geq 0.8)$ (Table 1$)$.

In comparison to lower SI, patients with elevated SI were 6 years younger ( $26 \pm 13$ vs $32 \pm 12.5, p=0.001)$, had lower PP $(41.3 \pm 16$ vs $52 \pm 16, p=0.001)$, lower GCS (11 \pm 4 vs $14 \pm 1, p=0.001)$, lower TRISS $(0.9381 \pm 0.114$ vs $0.9822 \pm 0.045, p=0.001$ ), and lower hematocrit values. Patients with elevated SI also had elevated initial serum lactate levels (median; 3.4 vs. $2.5, p=0.001$ ), greater ISS $(22 \pm 12$ vs $15 \pm 8.0, p=0.001)$ and higher abdominal AIS $(2.7 \pm 0.9$ vs $2.4 \pm 0.7, p=0.001)$. The mean SI was relatively higher in those who had blunt in comparison to penetrating trauma $(0.89 \pm 0.36$ vs $0.79 \pm 0.30)$.

Table 2 compares the associated injuries, hospital course and outcome by SI. The two groups were comparable for the reported SOI and hemothorax $(p>0.05$ for all). The elevated SI group showed higher association with head injury (35.6\% vs. $16.6 \%, p=0.001)$ and retroperitoneal hematoma $(10.4 \%$ vs. $4.2 \%, p=0.005)$. The rate of exploratory laparotomy $(33.6 \%$ vs. $23.7 \%, p=$ $0.009)$, blood transfusion $(60.2 \%$ vs. $22.6 \%, p=0.001)$ and MTP activation ( $21.8 \%$ vs. $3.9 \%, p=0.001)$ were significantly higher in patients with elevated SI. Figure 1 shows the study design and outcome.

Amongst the different injured organ, SI values varied and was higher in the injured organ in comparison to the non-injured organ; for example: it was $0.94 \pm 0.35$ in pancreatic injury vs $0.88 \pm 0.36$ in non-injured pancreas), $0.92 \pm 0.41$ in renal injury vs $0.87 \pm 0.34$ in non-injured kidney, $0.91 \pm 0.37$ in splenic injury vs $0.86 \pm 0.35$ in nonsplenic injury and $0.89 \pm 0.36$ in hepatic injury vs $0.89 \pm$ 0.35 in non-hepatic injury). Figure 2 shows that the median SI increased with the grade of liver and spleen injuries.

A significantly longer median ICU and hospital length of stay were found among patients with $\mathrm{SI} \geq 0.8$ than those with SI $<0.8$. Likewise, the proportions of sepsis (9.7\% vs $2.5 \%)$ and hospital death (14.2\% vs $3.2 \%)$ were significantly higher in patients with $\mathrm{SI} \geq 0.8$ (almost 4 times) when compared to those with $\mathrm{SI}<0.8$.

Table 3 demonstrates a significant positive and negative correlation between SI and other predictors.

Table 4 shows the performance and accuracy of different SI values $(\geq 0.8, \geq 0.9$, and $\geq 1.0)$ as well as the $\mathrm{ABC}$ score with respect to mortality, blood transfusion, MTP and exploratory laparotomy. SI $\geq 0.8$ had a higher sensitivity ( 85 and $82 \%$ ) and negative predictive value (96 and $97 \%$ ) with a negative LR of 0.27 and 0.34 to identify the need for MTP and risk of mortality, respectively.

For prediction of the need for exploratory laparotomy, the 3 SI values $(\geq 0.8, \geq 0.9$, and $\geq 1.0)$ and $A B C$ score showed a similar NPV (77\%) with poor sensitivity 
Table 1 Clinical characteristics, presentation and outcome by shock index in abdominal trauma patients sustained solid organ injuries

\begin{tabular}{|c|c|c|c|c|}
\hline & $\begin{array}{l}\text { Overall } \\
(n=572)\end{array}$ & $\begin{array}{l}\mathrm{SI}<0.8 \\
(n=283)\end{array}$ & $\begin{array}{l}S I \geq 0.8 \\
(n=289)\end{array}$ & $P$ value \\
\hline Age (mean $\pm S D)$ & $29.2 \pm 13.0$ & $32.4 \pm 12.5$ & $26.1 \pm 12.9$ & 0.001 \\
\hline Males & 509 (89.0\%) & $263(92.9 \%)$ & $246(85.1 \%)$ & 0.003 \\
\hline Blunt trauma & 543 (94.9\%) & $264(93.3 \%)$ & $279(96.5 \%)$ & \multirow{2}{*}{$\begin{array}{l}0.07 \\
\text { for all }\end{array}$} \\
\hline Penetrating injuries & 29 (5.1\%) & 19 (6.7\%) & $10(3.5 \%)$ & \\
\hline \multicolumn{5}{|l|}{ Mechanism of injury } \\
\hline Motor vehicle crash & 365 (77.7\%) & $170(73.6 \%)$ & $195(81.6 \%)$ & \multirow{2}{*}{$\begin{array}{l}0.03 \\
\text { for all }\end{array}$} \\
\hline Fall from height & 105 (22.3\%) & $61(26.4 \%)$ & 44 (18.4\%) & \\
\hline Pulse pressure ED & $46.6 \pm 16.6$ & $51.9 \pm 15.8$ & $41.3 \pm 15.6$ & 0.001 \\
\hline Initial lactate & $2.9(0.6-23.4)$ & $2.48(0.8-23.4)$ & $3.4(0.6-22.5)$ & 0.001 \\
\hline Second lactate & $3.0(0.7-80.0)$ & $2.7(0.7-39.0)$ & $3.2(0.8-80.0)$ & 0.03 \\
\hline Initial hematocrit & $39.6 \pm 7.0$ & $41.2 \pm 6.0$ & $38.0 \pm 7.6$ & 0.001 \\
\hline Second hematocrit & $36.6 \pm 6.45$ & $37.9 \pm 5.7$ & $35.3 \pm 7.0$ & 0.001 \\
\hline GCS ED & $12.4 \pm 2.6$ & $13.9 \pm 1.1$ & $10.9 \pm 4.1$ & 0.001 \\
\hline Injury Severity Score & $18.7 \pm 10.7$ & $15.0 \pm 8.1$ & $22.2 \pm 11.7$ & 0.001 \\
\hline Abdomen AIS & $2.6 \pm 0.9$ & $2.4 \pm 0.7$ & $2.7 \pm 0.9$ & 0.001 \\
\hline TRISS & $0.9618 \pm 0.0873$ & $0.9822 \pm 0.0457$ & $0.9381 \pm 0.1143$ & 0.001 \\
\hline
\end{tabular}

Shock Index (SI) = pulse ED / SBP ED; Pulse pressure = SBP ED - DBP ED; GCS: Glasgow Coma score

Table 2 Associated injuries and outcome by shock index

\begin{tabular}{|c|c|c|c|c|}
\hline & Overall $(n=572)$ & SI $<0.8(n=283)$ & $\mathrm{SI} \geq 0.8(n=289)$ & $P$ value \\
\hline \multicolumn{5}{|l|}{ Solid Organ injury ${ }^{a}$} \\
\hline Liver & $289(50.5 \%)$ & $143(50.5 \%)$ & $146(50.5 \%)$ & 0.99 \\
\hline Splenic & $211(36.9 \%)$ & $97(34.3 \%)$ & 114 (39.4\%) & 0.20 \\
\hline Kidney & $122(21.3 \%)$ & $59(20.8 \%)$ & $63(21.8 \%)$ & 0.78 \\
\hline Pancreas & $32(5.6 \%)$ & $12(4.2 \%)$ & $20(6.9 \%)$ & 0.16 \\
\hline \multicolumn{5}{|l|}{ Associated injuries } \\
\hline Head injury & $150(26.2 \%)$ & $47(16.6 \%)$ & 103 (35.6\%) & 0.001 \\
\hline Hemothorax & $53(9.3 \%)$ & $24(8.5 \%)$ & $29(10.0 \%)$ & 0.52 \\
\hline Retroperitoneal hematoma & $42(7.3 \%)$ & $12(4.2 \%)$ & 30 (10.4\%) & 0.005 \\
\hline Pelvic hematoma & $16(4.2 \%)$ & $5(2.7 \%)$ & $11(5.7 \%)$ & 0.14 \\
\hline Mesenteric injury & $53(9.7 \%)$ & 27 (9.9\%) & $26(9.4 \%)$ & 0.83 \\
\hline FAST Positive & $147(27.8 \%)$ & $61(23.6 \%)$ & $86(31.7 \%)$ & 0.03 \\
\hline Intubation & $209(36.5 \%)$ & $48(17.0 \%)$ & $161(55.7 \%)$ & 0.001 \\
\hline Exploratory laparotomy & $164(28.7 \%)$ & $67(23.7 \%)$ & 97 (33.6\%) & 0.009 \\
\hline Blood transfusion & $238(41.6 \%)$ & $64(22.6 \%)$ & $174(60.2 \%)$ & 0.001 \\
\hline Blood unit transfused & $6(1-62)$ & $4(1-62)$ & $6.5(1-51)$ & 0.001 \\
\hline MTP activation & 74 (12.9\%) & 11 (3.9\%) & $63(21.8 \%)$ & 0.001 \\
\hline ICU LOS & $4(1-76)$ & $3(1-76)$ & $5(1-69)$ & 0.003 \\
\hline Ventilatory days & $3(1-32)$ & $2(1-32)$ & $3(1-31)$ & 0.48 \\
\hline Hospital LOS & $8(1-304)$ & $6(1-122)$ & $10(1-304)$ & 0.001 \\
\hline Sepsis & $35(6.1 \%)$ & 7 (2.5\%) & $28(9.7 \%)$ & 0.001 \\
\hline In-hospital mortality & $50(8.7 \%)$ & $9(3.2 \%)$ & 41 (14.2\%) & 0.001 \\
\hline
\end{tabular}

${ }^{a}$ The frequency of each solid organ is overlapping between the four organs; SI Shock Index, MTP Massive transfusion protocol, LOS Length of stay 


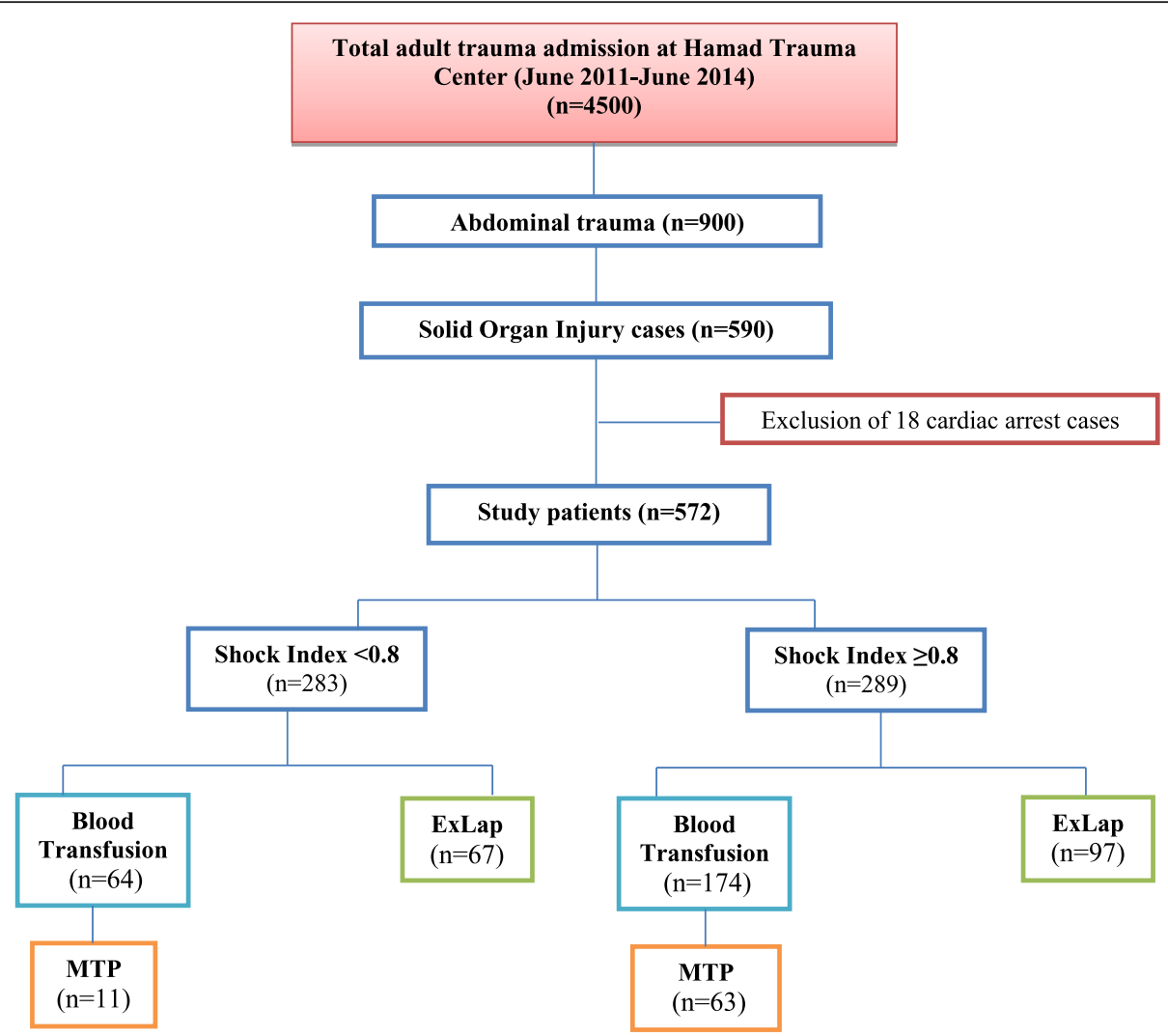

Fig. 1 Flow chart of the study design (MTP: Massive Transfusion Protocol; ExLap: Exploratory laparotomy)

(32-59\%), however, ABC score showed a higher specificity $(93 \%)$ and PPV of $63 \%$.

Figure 3 shows the ROC curves for different shock index (SI) cut-offs for prediction of blood transfusion in SOI. The AUC for SI $\geq 0.70$ is $0.62(0.56-0.69)$ and 0.71 (0.66-0.77) for SI $\geq 0.80$ ( $p=0.001$ for each). Different SI cut-offs $(<0.30,0.30-0.40,0.41-0.50,0.51-0.60,0.61-$ $0.70, \quad 0.71-0.80, \quad 0.81-0.90$, and $>0.90)$ were plotted against blood transfusion and MTP (Fig. 4). The need for blood transfusion and protocol increased significantly with SI 0.8 and above.

\section{Multivariable logistic regression analysis}

Multivariable analysis using 8 relevant variables such as age, sex, initial serum lactate, initial hematocrit value, FAST positivity, abdominal AIS, ISS, ED GCS and SI, it showed that showed that SI $\geq 0.8$ was an independent predictor of blood transfusion with OR 2.80; $95 \%$ CI 1.560-4.950). When SI was introduced as a continuous variable, the OR for blood transfusion was 15.00 (95\% CI 4.180-53.16).

For the prediction of MTP activation, multivariable regression analysis using 8 covariates showed that $\mathrm{SI} \geq 0.8$ was independent predictor of MTP with OR 2.81 (95\% CI 1.098-7.206). When SI was introduced as a continuous variable, the OR for blood transfusion was 1.41 (95\% CI 0.540-3.694).

For early exploratory laparotomy, SI was not predictor of laparotomy (OR 1.23; 95\% CI 0.689-2.212) (Table 5).

\section{Discussion}

The present study has several key findings. SI $\geq 0.8$ is a useful bedside simple predictor for early management of massive bleeding including the MTP activation in patients with SOI. Multivariate analysis model failed to address the role of SI in predicting the need of exploratory laparotomy; however, it showed that SI was independent predictor of blood transfusion and MTP. Moreover, the study showed significant correlations between SI and $\mathrm{ABC}$ scoring, serum lactate, the amount of transfused blood, and ICU/ hospital length of stay. Furthermore, patients with SI $\geq 0.8$ were found to have a higher rate of sepsis and in-hospital mortality in our cohort. Moreover, the higher the grade of organ injury, the higher the SI is in patients with liver and splenic injury. Therefore, SI could be used for early identification of SOI patients who are at risk of significant bleeding that requires massive transfusion. The AUC showed the superiority of SI 0.80 over the 0.70 cutoff for the prediction of blood transfusion in the present study. 

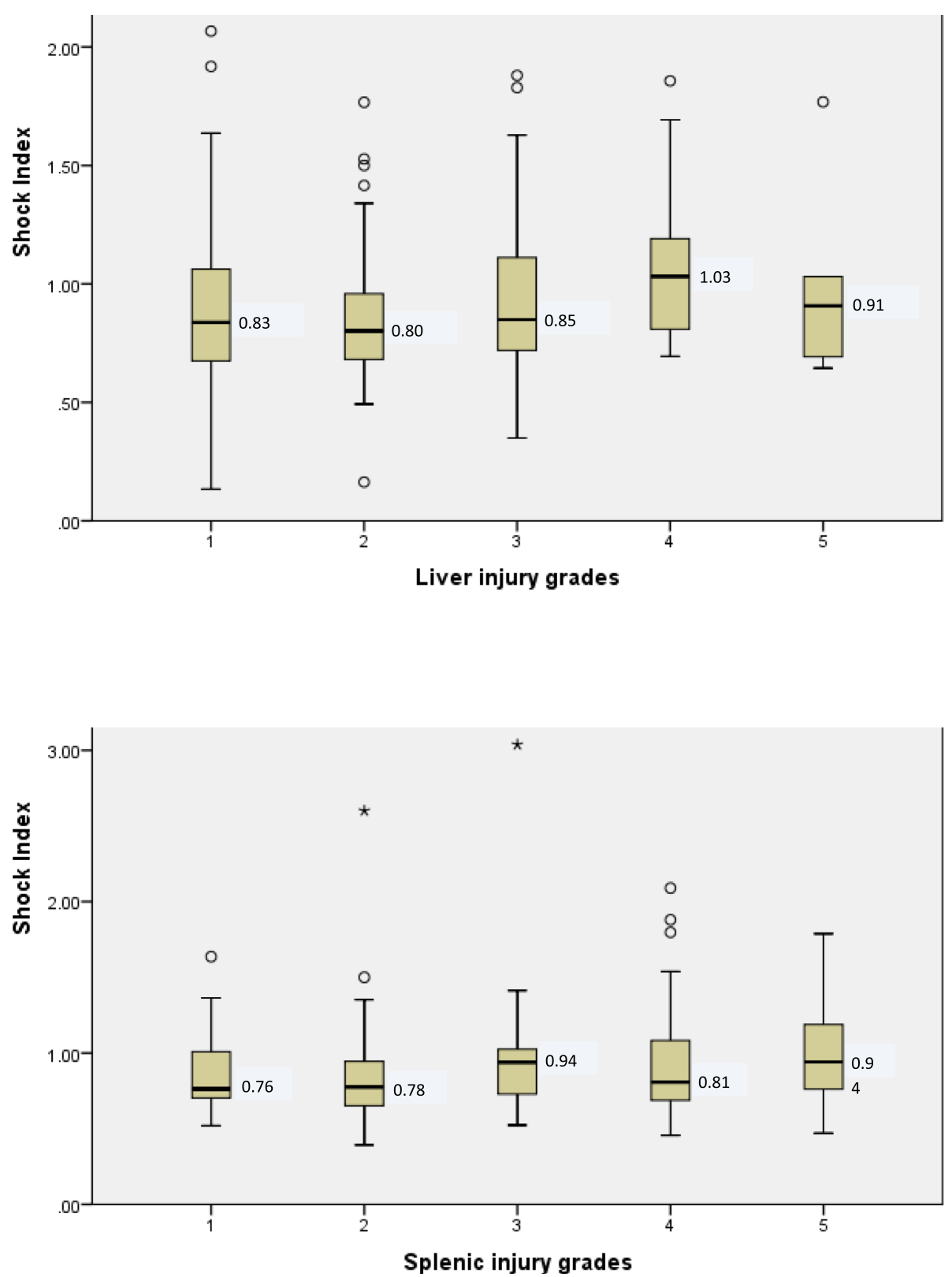

Fig. 2 Shock Index by (a) liver and (b) splenic injury grades

The present study explores the prognostic implications of SI in terms of blood transfusion, MTP activation and exploratory laparotomy in patients with traumatic SOI. Several investigators have proposed different cut-off values for SI, of which SI $\geq 0.8$, SI $\geq 0.9$ or $\geq 1.0$ has been used to predict critical bleeding in trauma patients (Cannon et al. 2009; El-Menyar et al. 2018; Schroll et al. 2018; Olaussen et al. 2014; Odom et al. 2016). The present study utilized $\mathrm{SI} \geq 0.8$ as this cut-off has higher sensitivity and NPV for prediction of blood transfusion. An earlier study suggested that the frequently used cutoff value of 0.9 has greater possibility of under-triage in patients necessitating urgent intervention (McNab et al. 2012).

Our institution is following the standard international management guidelines to treat patients with multiple trauma; many of these patients require blood transfusion $(\approx 42 \%)$ and one-third of them necessitate MTP activation. Caring for such patients is resource-intensive task and requires specialized coordinated services in a critical and timely manner (Peralta et al. 2015).

Exploratory laparotomy was required in one-quarter of cases that sustained blunt trauma. The initial FAST 
Table 3 Correlations between Sl, clinical parameters and injury severity

\begin{tabular}{lll}
\hline & Pearson correlation $(r)$ & $p$ value \\
\hline Age & -0.25 & 0.001 \\
Pulse pressure & -0.45 & 0.001 \\
Hematocrit & -0.34 & 0.001 \\
Serum lactate & 0.34 & 0.001 \\
GCS ED & -0.40 & 0.001 \\
Injury Severity score & 0.35 & 0.001 \\
Abdominal AlS & 0.15 & 0.001 \\
ABC score & 0.62 & 0.001 \\
TRISS & -0.24 & 0.001 \\
Blood units transfused & 0.22 & 0.001 \\
Hospital LOS & 0.23 & 0.001 \\
\hline
\end{tabular}

examination in our series yielded negative results in $72 \%$ of the blunt and $75 \%$ of the penetrating trauma. In those who had negative FAST results, blood transfusion, MTP activation and early laparotomy were required in 35,16 and $9 \%$ of cases, respectively.

On multivariate analysis, both high SI and positive FAST was almost having equal predictive value for transfusion and MTP. Although FAST has important diagnostic in the initial assessment of trauma patients, SI is an easy and early predictor that doesn't require an operator skill.

A systematic review (Bruijns et al. 2013) demonstrated that SI could be a better criterion for early identification of ongoing hemorrhage, when compared to separated vital signs i.e., heart rate (HR) and systolic blood pressure (SBP) alone. Heidar et al. (Heidar et al. 2014) showed a significant association between baseline SI or SBP and the need for surgical intervention in patients with blunt abdominal trauma. However, the investigators

Table $\mathbf{4}$ Predictive value of scoring systems

\begin{tabular}{|c|c|c|c|c|}
\hline & MTP & Blood transfusion & Exploratory Laparotomy & Mortality \\
\hline \multicolumn{5}{|l|}{ Shock Index $(\geq 0.8)$} \\
\hline Sensitivity & $85.1 \%$ & $73.1 \%$ & $59.1 \%$ & $82 \%$ \\
\hline Specificity & $54.6 \%$ & $65.6 \%$ & $52.9 \%$ & $52.5 \%$ \\
\hline Positive Predictive value & $21.8 \%$ & $60.2 \%$ & $33.6 \%$ & $14.2 \%$ \\
\hline Negative Predictive value & $96.1 \%$ & $77.4 \%$ & $76.3 \%$ & $96.8 \%$ \\
\hline Positive likelihood ratio & 1.87 & 2.12 & 1.25 & 1.72 \\
\hline Negative likelihood ratio & 0.27 & 0.41 & 0.77 & 0.34 \\
\hline \multicolumn{5}{|l|}{ Shock Index $(\geq 0.9)$} \\
\hline Sensitivity & $75.7 \%$ & $59.7 \%$ & $46.3 \%$ & $70 \%$ \\
\hline Specificity & $70.5 \%$ & $81.7 \%$ & $68.9 \%$ & $67.8 \%$ \\
\hline Positive Predictive value & $27.6 \%$ & $70 \%$ & $37.4 \%$ & $17.2 \%$ \\
\hline Negative Predictive value & $95.1 \%$ & $74 \%$ & $76.2 \%$ & $95.9 \%$ \\
\hline Positive likelihood ratio & 2.56 & 3.26 & 1.48 & 2.17 \\
\hline Negative likelihood ratio & 0.34 & 0.49 & 0.77 & 0.44 \\
\hline \multicolumn{5}{|l|}{ Shock Index ( $\geq 1.0)$} \\
\hline Sensitivity & $63.5 \%$ & $47.9 \%$ & $36 \%$ & $62 \%$ \\
\hline Specificity & $79.1 \%$ & $88.9 \%$ & $77.5 \%$ & $77 \%$ \\
\hline Positive Predictive value & $31.1 \%$ & $75.5 \%$ & $39.1 \%$ & $20.5 \%$ \\
\hline Negative Predictive value & $93.6 \%$ & $70.5 \%$ & $75.1 \%$ & $95.5 \%$ \\
\hline Positive likelihood ratio & 3.03 & 4.31 & 1.6 & 2.69 \\
\hline Negative likelihood ratio & 0.46 & 0.58 & 0.82 & 0.49 \\
\hline \multicolumn{5}{|l|}{ ABC Score } \\
\hline Sensitivity & $44.9 \%$ & $28.1 \%$ & $32 \%$ & $45.5 \%$ \\
\hline Specificity & $90.2 \%$ & $95.7 \%$ & $92.6 \%$ & $88.5 \%$ \\
\hline Positive Predictive value & $40.8 \%$ & $82.9 \%$ & $63.2 \%$ & $26.3 \%$ \\
\hline Negative Predictive value & $91.6 \%$ & $64.5 \%$ & $77.5 \%$ & $94.7 \%$ \\
\hline Positive likelihood ratio & 4.58 & 6.53 & 4.32 & 3.95 \\
\hline Negative likelihood ratio & 0.61 & 0.75 & 0.73 & 0.61 \\
\hline
\end{tabular}




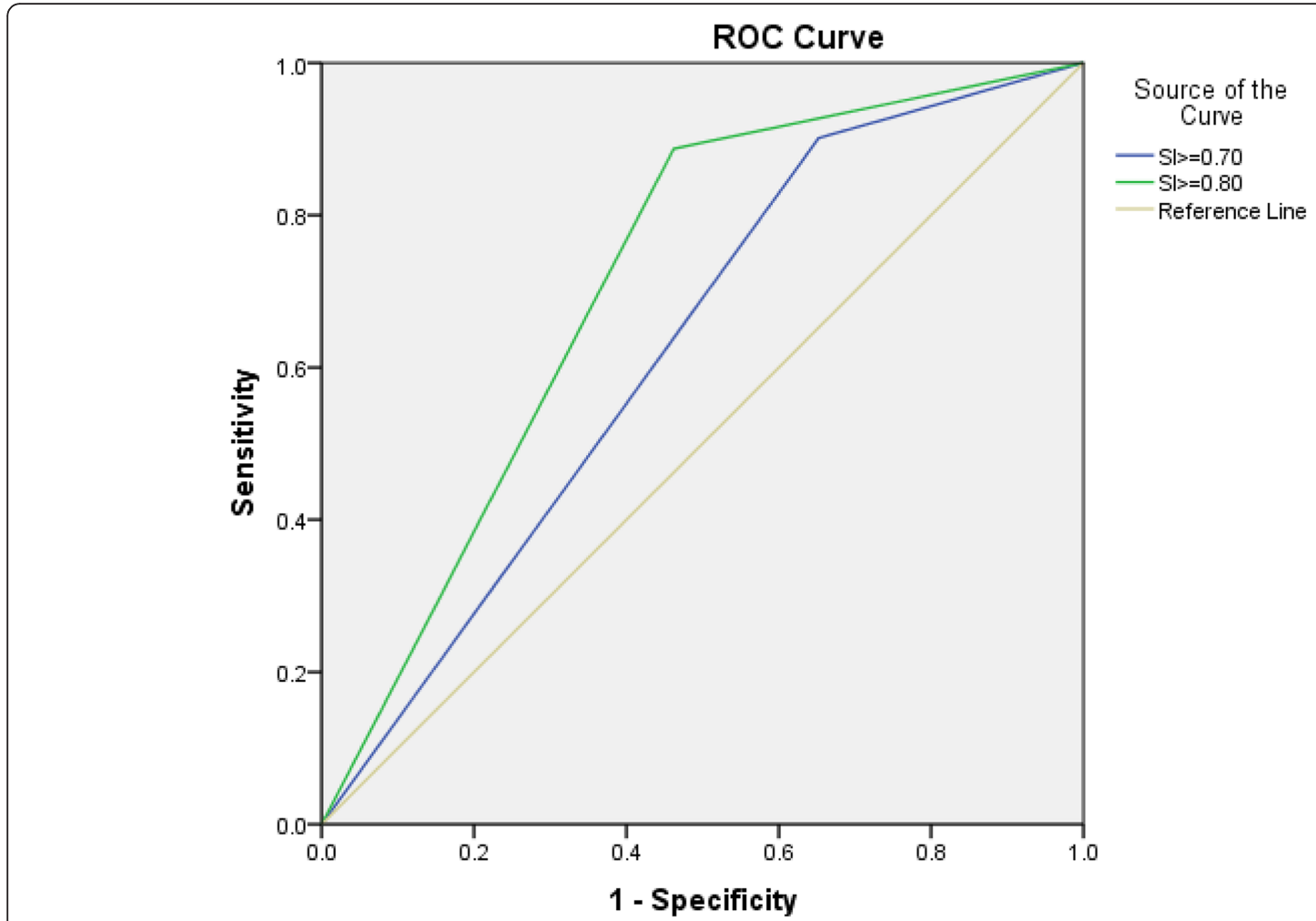

Fig. 3 ROC curves for different shock index (SI) cut-offs for prediction of blood transfusion in solid organ injury. Area under the Curve (AUC) for SI $\geq 0.70$ is $0.62(0.56-0.69)$ and $0.71(0.66-0.77)$ for $\mathrm{SI} \geq 0.80$

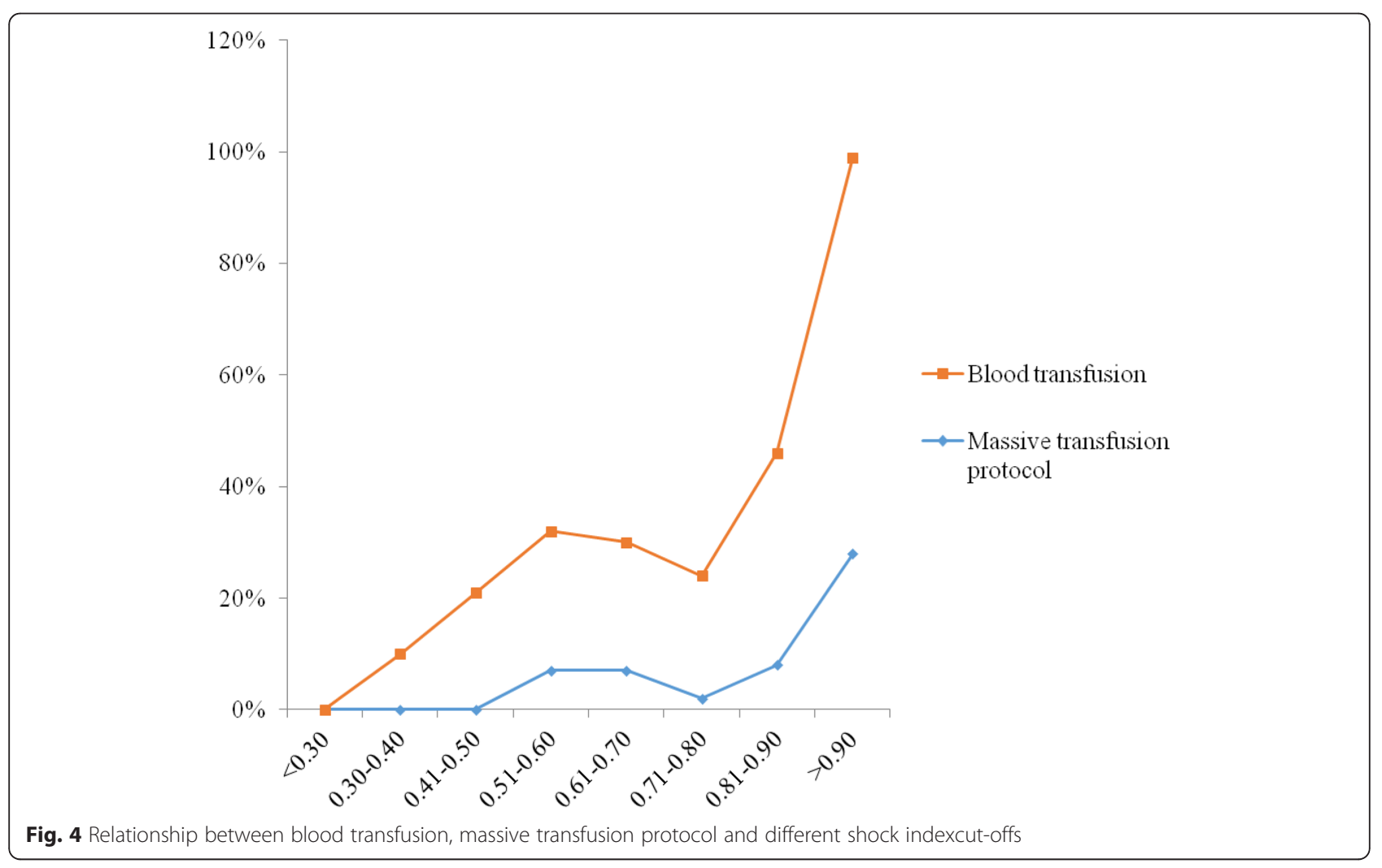


Table 5 Multivariable analysis for predictor of blood transfusion, massive transfusion protocol and laparotomy

\begin{tabular}{|c|c|c|c|c|c|c|c|c|c|}
\hline \multirow[t]{2}{*}{ Variable } & \multicolumn{3}{|c|}{ Blood transfusion } & \multicolumn{3}{|c|}{ Massive transfusion protocol } & \multicolumn{3}{|c|}{ Exploratory laparotomy } \\
\hline & $P$ & OR & $95 \% \mathrm{Cl}$ & $\mathrm{P}$ & OR & $95 \% \mathrm{Cl}$ & $P$ & OR & $95 \% \mathrm{Cl}$ \\
\hline Age; years* & 0.187 & 1.016 & $0.992-1.040$ & 0.137 & 1.025 & $0.992-1.059$ & 0.151 & 1.02 & $0.994-1.040$ \\
\hline Sex (male) & 0.318 & 1.649 & $0.618-4.398$ & 0.066 & 4.268 & $0.908-20.05$ & 0.136 & 2.18 & $0.783-6.047$ \\
\hline Initial Lactate* & 0.001 & 1.418 & $1.179-1.704$ & 0.166 & 1.09 & $0.966-1.226$ & 0.116 & 1.09 & $0.977-1.233$ \\
\hline Initial hematocrit* & 0.001 & 0.862 & $0.816-0.911$ & 0.001 & 0.884 & $0.833-0.938$ & 0.009 & 0.94 & $0.900-0.985$ \\
\hline Admission GCS* & 0.015 & 0.913 & $0.849-0.983$ & 0.010 & 0.897 & $0.826-0.975$ & 0.015 & 0.92 & $0.854-0.983$ \\
\hline ISS* & 0.001 & 1.072 & $1.032-1.114$ & 0.183 & 1.032 & $0.985-1.080$ & 0.003 & 0.94 & $0.911-0.981$ \\
\hline FAST result & 0.016 & 2.266 & $1.168-4.397$ & 0.079 & 2.24 & $0.910-5.532$ & 0.001 & 4.14 & $2.347-7.452$ \\
\hline Abdomen AIS* & 0.020 & 1.659 & $1.082-2.544$ & 0.172 & 1.40 & $0.862-2.295$ & 0.001 & 2.53 & $1.719-3.732$ \\
\hline $\mathrm{SI}{ }^{*}$ & 0.001 & 15.00 & $4.180-53.16$ & 0.482 & 1.41 & $0.540-3.694$ & 0.758 & 0.87 & $0.372-2.057$ \\
\hline SI ** & 0.001 & 2.80 & $1.560-4.950$ & 0.031 & 2.81 & $1.098-7.206$ & 0.479 & 1.23 & $0.689-2.212$ \\
\hline
\end{tabular}

$*=$ continuous variable, ${ }^{* *}$ Shock index (SI) as categorical variable $\geq 0.80$ vs $<0.80$ in a second multivariable model, OR Odd ratio, $\mathrm{Cl}$ Confidence interval

found that the baseline HR was comparable in the surgical and conservative groups. Several scoring systems have been proposed for identification of post-traumatic hemorrhage and MT, however, most of them seemed to be complicated, resource-intensive, not validated, not immediately available, or time consuming (Callcut et al. 2016; DeMuro et al. 2013). Vandromme et al. (Vandromme et al. 2011) grouped their study cohort into 6 categories based on SI. The authors observed a linear relationship between SI and blood requirements with a 1.6 fold increased odds of massive transfusion for $\mathrm{SI}>0.9-1.1$ which substantially increased to 5.57 fold in patients with greater SI (>1.1-1.3). This finding showed that SI is sensitive to changes in the circulating blood volume, and is useful in accurately predicting the need for early intervention to stop ongoing bleeding (Birkhahn et al. 2005).

To date, several approaches have been proposed to detect the extent of hypovolemic shock during early hemostatic resuscitation in trauma patients with variable applicability (Mutschler et al. 2013; Brockamp et al. 2012). In a validation study of 6 scoring systems for the need of MT after trauma, the greater overall precision was identified for the Trauma-Associated Severe Hemorrhage (TASH) score and Prince of Wales Hospital/Rainer (PWH) score (Brockamp et al. 2012). In addition, the Traumatic Bleeding Severity Score (TBSS) has been introduced to accurately predict the need for MT, but it had a sophisticated calculation that limits its use in the pre-hospital settings and/or upon hospital admission (Ogura et al. 2014). The ABC scoring system does not involve laboratory tests in its calculation and provides valuable information for the blood requirements in critical conditions. However, it requires FAST result which restricts its ready availability on ED admission except in well-designed ED of trauma centers where ultrasound machines are immediately available at bed side. Also, the use of other scoring systems that need laboratory tests and cumbersome calculations is inapplicable in emergency situations (Vandromme et al. 2011; El-Menyar et al. 2019). Recently, Motameni et al. concluded that in comparison to the clinical evaluation, the $\mathrm{ABC}$ criteria may overestimate the need for MT and it may even increase the product wastage, however, it could lead to earlier MTP activation (Motameni et al. 2018). In our study, ABC scores showed a significant correlation with SI $(r=0.62)$, a finding that supports the importance of SI as a simple alternative predictor in patient with SOI.

\section{Limitations}

Our study has potential limitations due to its retrospective nature, and thus there is a possibility of missing information and inherent selection bias. Data were collected from a single center which would affect, in addition to the previous factors, the generalizability of our results. We focused on the SOI as it is a main source of bleeding in traumatic abdominal injury. The administration of blood products and massive transfusion is based on clinical judgment rather than on the objective measurement of hemorrhage. We lack information regarding the use of home medications (i.e., beta blockers) which could also influence SBP and HR; however, we would not expect to find frequent comorbidities because our patients are young with a mean age 29 years. Females constituted only one tenth of the study cohort whereas the vast majority (89\%) was males; therefore the influence of gender disparity in trauma care was not discussed. The vital signs and the SI used in this study were based on initial values; we did not have detailed information regarding the exact timing of measurement of vital signs apart from the fact that these were measured as initial vital signs (within the first 5 min post arrival). Findings based on a single measurement may differ from the average of multiple readings. Assessment of the SI at ED admission might be influenced by the pre-hospital care involving the administration of intravenous fluids, 
sedation, and/or the use of vasopressors. Pain and anxiety might also have an influence on SBP and HR and therefore on the SI. There was no specific prehospital protocol, different than the international consensus, to follow. We did not report on the EMS transportation time that may affect the initial vital signs on arrival, however, based on our previous work the median EMS time was 58 min (interquartile range $45-77 \mathrm{~min}$ ) (Al-Thani et al. 2014). This study comprised of mainly blunt trauma (95\%) cases; the cardiovascular responses in patients with blunt trauma may differ from those with penetrating injuries. Although SI is also a useful tool for triaging patients for improved outcomes and effective utilization of resources (El-Menyar et al. 2017; Khan et al. 2013; Heidar et al. 2014), we do believe that further prospective studies comparing utility of SI with the other scoring tools are needed in patients with potential SOI.

\section{Conclusions}

Shock index is a simple, fast, and bedside physiological tool that can predict the need for MTP activation in blunt abdominal trauma, particularly in patient who sustained SOI who can benefit from early massive transfusion and intervention. Further prospective studies are needed to support our findings and to compensate for our study limitations.

\section{Abbreviations}

FAST: Focused abdominal sonography assessment; MTP: Massive blood transfusion protocol; SI: Shock index; SOI: Solid organ injury

\section{Acknowledgments}

The authors thank all the staff of the research office as well as the trauma registry at the trauma surgery section, Department of surgery.

\section{Authors' contributions}

$A E, G J, M A, H A, I M$, and HA have Substantial contributions to the acquisition, analysis, or interpretation of data for the work; drafting the work or revising it critically for important intellectual content; and final approval of the version to be published.

\section{Funding}

This research did not receive any specific grant from any funding agency.

\section{Availability of data and materials}

Not applicable

\section{Ethics approval and consent to participate}

This study obtained ethical approval from Research Ethics Committee, at Medical Research Center, Hamad Medical Corporation (HMC), Doha, Qatar (IRB\#14409/14) with a waiver of consent. No direct contact with participants and data were collected anonymously.

\section{Consent for publication}

Research Ethics Committee, at Medical Research Center, Hamad Medical Corporation (HMC), Doha, Qatar (IRB\#14409/14).

\section{Competing interests}

The authors declare that they have no competing interests.

\section{Author details}

${ }^{1}$ Clinical Medicine, Weill Cornell Medical College, Doha, Qatar. ${ }^{2}$ Department of Surgery, Clinical Research, Trauma \& Vascular Surgery, Hamad General
Hospital (HGH), P.O Box 3050, Doha, Qatar. ${ }^{3}$ Department of Surgery, Trauma Surgery, HGH, Doha, Qatar. ${ }^{4}$ Department of Surgery, Trauma \& Vascular Surgery, HGH, Doha, Qatar.

Received: 18 June 2019 Accepted: 28 August 2019

Published online: 07 October 2019

\section{References}

Al-Thani H, El-Menyar A, Latifi R. Prehospital versus emergency room intubation of trauma patients in Qatar: A-2-year observational study. N Am J Med Sci. 2014;6(1):12-8

Arumugam S, Al-Hassani A, El-Menyar A, et al. Frequency, causes and pattern of abdominal trauma: a 4-year descriptive analysis. J Emerg Trauma Shock. 2015; 8:193-8.

Birkhahn RH, Gaeta TJ, Terry D, et al. Shock index in diagnosing early acute hypovolemia. Am J Emerg Med. 2005;23:323-6.

Brockamp T, Nienaber U, Mutschler M, et al. Predicting on-going hemorrhage and transfusion requirement after severe trauma: a validation of six scoring systems and algorithms on the TraumaRegister DGUOे. Crit Care. 2012;16: R129.

Bruijns SR, Guly HR, Bouamra O, et al. The value of traditional vital signs, shock index, and age-based markers in predicting trauma mortality. J Trauma Acute Care Surg. 2013;74:1432-7.

Callcut RA, Cripps MW, Nelson MF, et al. The massive transfusion score as a decision aid for resuscitation: learning when to turn the massive transfusion protocol on and off. J Trauma Acute Care Surg. 2016;80:450-6.

Cannon CM, Braxton CC, Kling-Smith M, et al. Utility of the shock index in predicting mortality in traumatically injured patients. J Trauma. 2009;67:1426-30.

DeMuro JP, Simmons S, Jax J, Gianelli SM. Application of the shock index to the prediction of need for hemostasis intervention. Am J Emerg Med. 2013;31: $1260-3$.

El-Menyar A, Abdelrahman H, Al-Hassani A, et al. Single versus multiple solid organ injuries following blunt abdominal trauma. World J Surg. 2017;41: 2689-96.

El-Menyar A, Goyal P, Tilley E, et al. The clinical utility of shock index to predict the need for blood transfusion and outcomes in trauma. J Surg Res. 2018; 227:52-9.

El-Menyar A, Mekkodathil A, Abdelrahman H, Latifi R, Galwankar S, Al-Thani H, Rizoli S. Review of existing scoring systems for massive blood transfusion in trauma patients: where do we stand? Shock. 2019. https://doi.org/10.1097/ SHK.0000000000001359.

Fredericks C, Kubasiak JC, Mentzer CJ, Yon JR. Massive transfusion: An update for the anesthesiologist. World J Anesthesiol. 2017;6(1):14-21. https://doi.org/10. 5313/wja.v6.i1.14

Heidar A, Ravanfar P, Namazi G, et al. Determinants of successful non-operative Management of Intra- Peritoneal Bleeding Following Blunt Abdominal Trauma. Bull Emerg Trauma. 2014;2:125-9.

Hsu Y, Haas T, Cushing M. Massive transfusion protocols: current best practice. Int J Clin Transfus Med. 2016:4:15-27. https://doi.org/10.2147/IJCTM.S61916.

Khan S, Allard S, Weaver A, et al. A major haemorrhage protocol improves the delivery of blood component therapy and reduces waste in trauma massive transfusion. Injury. 2013;44:587-92.

Malhotra AK, Ivatury RR, Latifi R. Blunt abdominal trauma: evaluation and indications for laparotomy. Scand J Surg. 2002;91:52-7.

McNab A, Burns B, Bhullar I, et al. A prehospital shock index for trauma correlates with measures of hospital resource use and mortality. Surgery. 2012;152:473-6.

McNab A, Burns B, Bhullar I, et al. An analysis of shock index as a correlate for outcomes in trauma by age group. Surgery. 2013;154:384-7.

Motameni AT, Hodge RA, McKinley WI, et al. The use of ABC score in activation of massive transfusion: the yin and the yang. J Trauma Acute Care Surg. 2018;85:298-302.

Mutschler M, Nienaber U, Münzberg M, et al. The Shock Index revisited - a fast guide to transfusion requirement? A retrospective analysis on 21,853 patients derived from the TraumaRegister DGU. Crit Care. 2013;17:R172. https://doi. org/10.1186/cc12851.

Odom SR, Howell MD, Gupta A, et al. Extremes of shock index predicts death in trauma patients. J Emerg Trauma Shock. 2016;9:103-6.

Ogura T, Nakamura Y, Nakano M, et al. Predicting the need for MT in trauma patients: the traumatic bleeding severity score. J Trauma Acute Care Surg. 2014;76:1243-50 
Olaussen A, Blackburn T, Mitra B, et al. Review article: shock index for prediction of critical bleeding post-trauma: a systematic review. Emerg Med Australas. 2014;26:223-8.

Peralta R, Vijay A, El-Menyar A, et al. Trauma resuscitation requiring massive transfusion: a descriptive analysis of the role of ratio and time. World J Emerg Surg. 2015;10:36. https://doi.org/10.1186/s13017-015-0028-3.

Rossaint R, Bouillon B, Cerny V, et al. The European guideline on management of major bleeding and coagulopathy following trauma: fourth edition. Crit Care. 2016;20:100.

Sawhney C, Kaur M, Gupta B, et al. Critical care issues in solid organ injury: review and experience in a tertiary trauma center. Saudi J Anaesth. 2014;8(Suppl 1): S29-35.

Schroll R, Swift D, Tatum D, et al. Accuracy of shock index versus ABC score to predict need for massive transfusion in trauma patients. Injury. 2018:49:15-9.

Stawicki SP. Trends in nonoperative management of traumatic injuries - a synopsis. Int J Crit IIIn Inj Sci. 2017;7:38-57.

Vandromme MJ, Griffin RL, Kerby JD, et al. Identifying risk for massive transfusion in the relatively normotensive patient: utility of the prehospital shock index. J Trauma. 2011;17:384-8.

Zarzaur BL, Croce MA, Fischer PE, et al. New vitals after injury: shock index for the young and age $x$ shock index for the old. J Surg Res. 2008;147:229-36.

\section{Publisher's Note}

Springer Nature remains neutral with regard to jurisdictional claims in published maps and institutional affiliations.

Ready to submit your research? Choose BMC and benefit from:

- fast, convenient online submission

- thorough peer review by experienced researchers in your field

- rapid publication on acceptance

- support for research data, including large and complex data types

- gold Open Access which fosters wider collaboration and increased citations

- maximum visibility for your research: over $100 \mathrm{M}$ website views per year

At $\mathrm{BMC}$, research is always in progress.

Learn more biomedcentral.com/submissions 